\title{
Discriminatory Impact of the COVID-19 Pandemic on Women Physicians
}

\author{
Sabita Jiwnani ${ }^{1}$ \\ ${ }^{1}$ Division of Thoracic Surgery, Department of Surgical Oncology, Tata \\ Memorial Centre, Homi Bhabha National Institute, Mumbai, \\ Maharashtra, India \\ Ind J Med Paediatr Oncol 2022;43:15-18.
}

\begin{abstract}
Address for correspondence Sabita Jiwnani, MCh, DNB, MRCS, Division of Thoracic Surgery, Department of Surgical Oncology, Tata Memorial Centre, Homi Bhabha National Institute, Mumbai 400012, Maharashtra, India

(e-mail: sabitajiwnani@gmail.com; jiwnanis@tmc.gov.in).
\end{abstract}

\begin{abstract}
Keywords

- childcare

- COVID-19

- domestic responsibilities

- women physicians

The impact of gender and women's traditional roles in society has been particularly pronounced during the coronavirus disease 2019 (COVID-19) pandemic. We conducted an online survey to evaluate the impact of the COVID-19 pandemic on domestic responsibilities among Indian physicians to look for gender-based differences by collecting data regarding domestic work and childcare, changes in work patterns, and lack of domestic help during the pandemic and the resultant nationwide lockdown. Through this study, we reported the results of a nationwide survey and showed how gender gaps exist, even within highly qualified professionals such as physicians, and how the COVID-19 pandemic has widened this gap. Our study showed statistically higher contribution by women physicians as compared to men not only in the increased domestic work, but also when it came to childcare and education. More women than men were forced to take additional leave and quit their jobs due to the increased domestic responsibilities.

In addition to highlighting the difficulties faced by the physician community as a whole due to nonavailability of domestic help and childcare facilities, this survey demonstrated the intense challenges women physicians face as they try to navigate the work-life balance dilemma by providing care to their families as well as patients. Our study highlighted the need to re-examine the specific challenges faced by women physicians and identify means to support and empower them.
\end{abstract}

The coronavirus disease 2019 (COVID-19) pandemic is likely to have a protracted effect on the lives of healthcare workers, especially women. Lockdowns across the world were a double whammy for most women physicians as they were not only being expected to fulfil their roles as frontline health workers but also as primary care providers at home in the absence of school, childcare, and domestic help. ${ }^{1}$ The increasing need for women to prioritize their families during this period has forced them to scale back their professional pursuits. $^{2}$ The pandemic brought into the limelight the preexisting gender disparities in their professional and personal lives, possibly reversing decades of efforts to bridge the gender gap and these effects are likely to be associated with long-term outcomes in the healthcare community. ${ }^{3}$

Gender imbalance is not a new topic of conversation in medicine. The number of women in medicine has grown
DOI https://doi.org/ 10.1055/s-0042-1742657. ISSN 0971-5851.

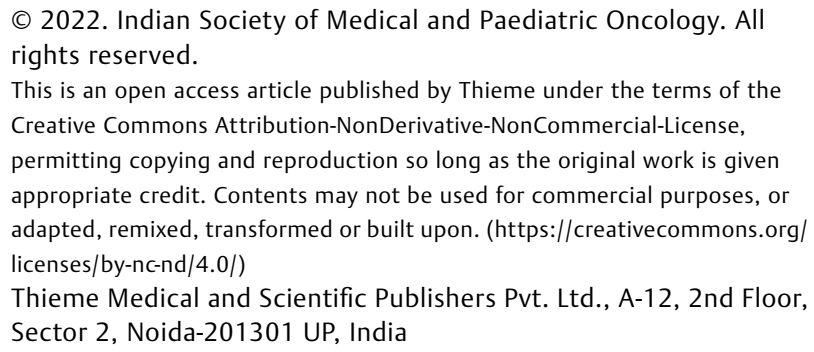


rapidly since 1970 . Half of medical school graduates in the United States are women and they comprise $38 \%$ of the faculty positions, but significant disparities exist in job achievement and compensation in medicine among male and female physicians. ${ }^{4,5}$ An analysis in 2014 evaluating more than 90,000 physicians found significant differences in faculty rank after adjustment for age, research, authorship, and experience. ${ }^{6}$

Although the number of women oncologists continues to increase, in a cross-sectional study by Chowdhary et al, evaluating 6,030 oncology faculty in 265 academic programs in the United States, women comprised $37 \%$ of faculty positions, but $21 \%$ of leadership positions in medical oncology, $12 \%$ in radiation oncology, and only $3.8 \%$ in surgical oncology. ${ }^{7}$

The first woman physician of India, Anandibai Joshi, graduated in 1886, but it took 125 years for young women opting for medical sciences to outnumber the male candidates, that is, in 2014-2015, the number of female candidates crossed $51 \%{ }^{8}$ However, this has not led to an increase in the female medical graduates actively practicing medicine, and less than one-third of the allopathic physicians in the country are women. ${ }^{9}$ Many factors have been attributed to this deficiency of women in the medical workforce, primarily being extended years of training, long working hours, and the necessity to manage work with domestic responsibilities. ${ }^{3}$

Women, especially mothers, regardless of employment status, have been the primary caregivers, multitasking and coping with the household chores as well as childcare and education, often alone with little or no support. Most urban households in India, especially those with working women, rely on extended family and domestic help, either part or full time, to navigate through their daily lives.

We conducted an online survey ${ }^{10}$ among Indian physicians in October 2020, after the easing of restrictions across India, to gather information on how the pandemic and subsequent lockdown had impacted the physician community and whether there were any differences according to gender. The survey was disseminated to a database of physicians, who had attended past evidence-based meetings and oncology conferences at a tertiary cancer care center, by email and a text messaging application. We designed this survey to try and understand the difficulties and challenges faced by physicians due to increased domestic work during this time. The 31-point questionnaire consisted of questions pertaining to the demographics, family setup, availability of domestic help, management of domestic chores, groceries, childcare, and children's education, both pre and post lockdown. We also asked questions regarding the impact of the pandemic and subsequent increase in the domestic responsibilities on professional duties. No formal written informed consent was taken to participate in the survey; completing the questionnaire implied consent. The survey was completely anonymous; no identifiable information was collected. The completed questionnaire was submitted online. The questionnaire was discontinued after 2 weeks of dissemination. The completed questionnaires were analyzed after receiving responses.
Our survey received 1,041 responses in 2 weeks of October 2020 with 62\% (643) male respondents. Most respondents, both men and women, were below the age of 50 years, approximately $70 \%$ in both groups. Considerably few trainees responded to the survey, with $81 \%$ of the female respondents and $86 \%$ of the male respondents being consultants. Among the various specialties, $45 \%$ of the respondents belonged to surgical and allied specialties, $14 \%$ belonging to pathology, and the rest to other specialties such as internal medicine, radiology, and pediatric as shown in - Fig. 1. Approximately $73 \%$ of the respondents worked in tertiary centers, almost half in private, and $66 \%$ had reduced working hours during the lockdown. Close to $90 \%$ of the respondents in both groups were married, with $81 \%$ having children and $70 \%$ of the respondents living in a joint family. Equal numbers of male and female respondents were married to healthcare workers (63 vs. 66\%) with $93 \%$ of women's spouses working during the lockdown versus $67 \%$ of men's spouses $(p<0.0001)$.

Domestic help was difficult to obtain during the lockdown, with $60 \%$ of the respondents not having any help during the severe lockdown between April and July 2020, while $83 \%$ of female respondents versus $78 \%$ of male respondents had domestic help before the lockdown $(p=0.056)$. Domestic responsibilities surged for $90 \%$ of the women versus $82 \%$ men. During the lockdown, $81 \%$ of the women handled the domestic chores and groceries either alone or with some help versus $63 \%$ of the men. More women (152/393) versus men $(123 / 520, p=0.00001)$ were solely responsible for the domestic chores, as shown in - Fig. 2.

When it came to childcare, our survey showed that prior to the lockdown male physician's spouses were primarily responsible for childcare in 112 out of 411 cases (25.9\%) compared to 17 out of 225 female physicians' spouses (7.5\%). During the complete shutdown, $61 \%$ of women looked after their children, either alone (16\%) or with help from other family members, versus only $32 \%$ of the men who participated in childcare responsibilities (-Fig. 2 ). The nonavailability of schools and childcare facilities led to the introduction to virtual education, which proved challenging for both children and parents. Also, $43 \%$ of the respondents felt that the children's educational burden had moderately increased, with $12.5 \%$ finding this escalation to be severe. Approximately three-fourth (74\%) of the women were responsible for child's educational activities along with some help from a family member compared to $31 \%$ of the men. Compared to men, women were more involved in their children's education solely $(2.5 \%$ men vs. $38 \%$ women, $p<0.01)$, as shown in -Fig. 2.

Although a majority of the physicians perceived that their work performance (clinical, teaching, administrative, research, alone or in combination) was impacted by the excessive domestic chores, this brunt was felt more acutely by women with $61 \%$ of the women experiencing a negative influence on work and 30\% feeling a moderate to severe reduction. Among male physicians, 369/643 did not feel any impact of the increased domestic responsibilities on their work performance compared to $154 / 393$ female physicians $(p<0.00001)$. An extreme impact of household obligations 


\section{What is your speciality}

\section{1,043 responses}

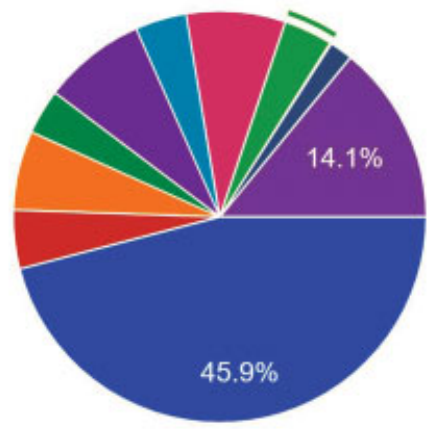

Surgery and allied fields (including EN...

Anesthesia

Medicine

Radiology

Pathology

Pediatric

Radiation oncology

Medical oncology

Fig. 1 Specialties of survey respondents.

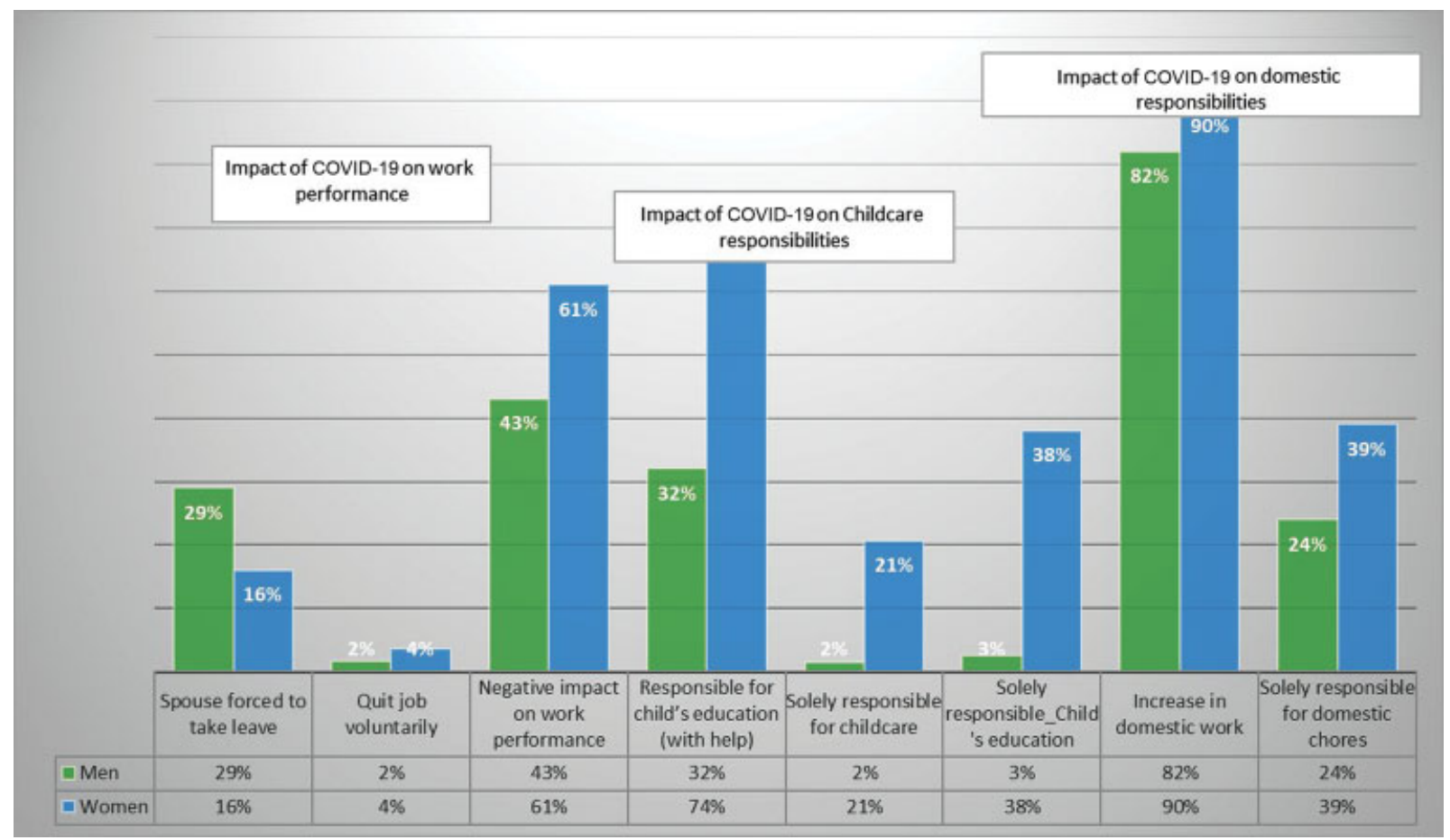

Fig. 2 Impact of coronavirus disease 2019 pandemic on domestic responsibilities, child care, and work.

was felt by $37 / 393$ women compared to $25 / 643$ men $(p=0.0002)$. Due to increased domestic responsibilities, fewer women's spouses (57/359) than men's (174/594, $p=0.00001$ ) had to forcibly take leave or work for reduced hours. About $3.5 \%$ of the women respondents versus 1.5\% men voluntarily quit their jobs, as shown in - Fig. 2. Almost $80 \%$ of both men and women felt they needed some flexibility or change in their working hours.

Our survey demonstrated that a disproportionately larger percentage of women physicians have been forced to shoulder domestic as well as childcare responsibilities, with the children's educational burden adding a significant toll. A similar survey was conducted recently in the United States with more than 2,700 respondents and the findings were presented at the Women in Medicine virtual summit. ${ }^{11}$ They found only $25 \%$ of the women physicians could continue to work without altering their schedules and less than half of the emergency medicine physician mothers felt adequately prepared for the pandemic, personally or professionally, more so those with young children.

Even in developed nations, employed women face challenges as they spend $22 \%$ more time on unpaid household and care work compared to men. ${ }^{12}$

Women have been forced to cut down on their work hours four to five times more than men due to childcare during the pandemic. ${ }^{1,13}$ Approximately $80 \%$ of the hospital employees are women, but no attempt has been made to recognize and solve the challenges being faced by them due to the onset of 
the pandemic. ${ }^{14}$ Thus women are not only over worked, but also risk facing a higher rate of burnout. The COVID-19 pandemic has magnified the challenges faced by working women in general, and physicians in particular.

A Harvard Business Review research showed that a gender diverse workforce is associated with increase in innovation and better productivity, with heightened influence and acceptability in a divergent community. ${ }^{15}$ Women physicians are an integral part of the healthcare community and they need support, not only from their male peers and families but also society at large, to retain their place in their professional environment.

Several strategies have been suggested to promote the retention of women, but there are limited data on impacts of interventions. Institutions helping employees recognize bias, fair compensation and provision of resources, satisfaction with mentorship, peer mentorship, and women role models within the institutions were associated with intent to remain at an institution. ${ }^{16} \mathrm{~A}$ gender diverse leadership in the medical field can also lead to increasing role models who can inspire graduating residents to pursue academic positions. The fragile Indian healthcare system needs innovative and specific interventions to retain and support the workforce constituted by the female physicians. Increased access to paid maternal as well as paternal leave, ability to reliably outsource domestic tasks, as well as in-campus options for childcare facilities and crèches are some of the practical solutions besides increased mentorship that can go a long way in boosting the confidence of the women physicians who are getting sidelined by the pandemic and getting relegated to outdated traditional roles as primary caregivers at home.

\section{Conflict of Interest}

None.

\section{References}

1 Brubaker L. Women physicians and the COVID-19 pandemic. JAMA 2020;324(09):835-836

2 Jones Y, Durand V, Morton K, et al; ADVANCE PHM Steering Committee. Collateral damage: how COVID-19 is adversely impacting women physicians. J Hosp Med 2020;15(08):507-509
3 Matulevicius SA, Kho KA, Reisch J, Yin H. Academic medicine faculty perceptions of work-life balance before and since the COVID-19 pandemic. JAMA Netw Open 2021;4(06):e2113539

4 Association of American Medical Colleges. US medical school applicants and students: 1982-1983 to 2011-2012. https://www.aamc.org/download/153708/data/ Published 2012. Accessed January 18, 2022

5 Richter KP, Clark L, Wick JA, et al. Women physicians and promotion in academic medicine. N Engl J Med 2020;383(22): $2148-2157$

6 Jena AB, Khullar D, Ho O, Olenski AR, Blumenthal DM. Sex differences in academic rank in US medical schools in 2014. JAMA 2015;314(11):1149-1158

7 Chowdhary M, Chowdhary A, Royce TJ, et al. Women's representation in leadership positions in academic medical oncology, radiation oncology, and surgical oncology programs. JAMA Netw Open 2020;3(03):e200708

8 Jayadev C, Sanjay S. Women in the "eye" of a pandemic!. Indian J Ophthalmol 2021;69(02):446-447

9 Rao KD, Bhatnagar A, Berman P. So many, yet few: human resources for health in India. Hum Resour Health 2012;10:19. Doi: 10.1186/1478-4491-10-19

10 Jiwnani S, Ranganathan P, Tiwari V, et al. COVID-19 pandemic and its gendered impact on Indian physicians. JCO Glob Oncol 2021; 7:1093-1100

11 Madani K, Pendergrast T, Sundareshan V, Jain S. The women in medicine summit: an evolution of empowerment in Chicago, Illinois, October 9 and 10, 2020: event highlights, scientific abstracts, and dancing with markers. Int J Academic Medicine. 2020;6(04):337-398

12 Hayes J, Hess C, Ahmed T. Institute for Women's Policy ResearchProviding unpaid household and care work in the United States: uncovering inequality. 2020https://iwpr.org/iwpr-publications/report/providing-unpaid-household-and-care-work-in-the-united-states-uncovering-inequality/ Accessed January 18, 2022

13 Collins C, Landivar LC, Ruppanner L, Scarborough WJ. COVID-19 and the gender gap in work hours. Gend Work Organ 2020;28(1 suppl):101-112

14 Kantamneni N. The impact of the COVID-19 pandemic on marginalized populations in the United States: a research agenda. J Vocat Behav 2020;119:103439

15 Harvard Business Review. Research: When Gender Diversity Makes Firms More Productive. 2019https://hbr.org/2019/02/research-when-gender-diversity-makes-firms-more-productive Accessed January 18, 2022

16 Onumah C, Wikstrom S, Valencia V, Cioletti A. What women need: a study of institutional factors and women faculty's intent to remain in academic medicine. J Gen Intern Med 2021;36(07): 2039-2047 